\title{
Experimental determination of step energies from island shape fluctuations: A comparison to the equilibrium shape method for $\mathrm{Cu}(100), \mathrm{Cu}(111)$, and $\mathrm{Ag}(111)$
}

\author{
Christoph Steimer, Margret Giesen, Laurens Verheij, and Harald Ibach* \\ Institut für Schichten und Grenzfächen ISG-3, Forschungszentrum Jülich, D-52425 Jülich, Germany
}

(Received 8 March 2001; revised manuscript received 4 May 2001; published 7 August 2001)

\begin{abstract}
Absolute values for step energies can be determined from the temperature dependence of island equilibrium shapes and the size dependence of island shape fluctuations. Experimental data on island fluctuations are evaluated and the resulting step energies are compared to those obtained earlier from the temperature dependence of the equilibrium shape. For islands on $\mathrm{Cu}(100), \mathrm{Cu}(111)$, and $\mathrm{Ag}(111)$, the step energies obtained by the two entirely different and independent methods agree within the experimental error. The advantages and disadvantages of the two methods are discussed.
\end{abstract}

DOI: 10.1103/PhysRevB.64.085416

PACS number(s): 68.35.Md, 05.70.Np, 68.03.Cd

\section{INTRODUCTION}

The free energy of monatomic steps on single crystal surfaces is a rather important energetic parameter. It determines the size and the roughening temperature of facets of the equilibrium shape of crystals. ${ }^{1-3}$ Minimization of the step free energy is the driving force for coarsening phenomena at surfaces such as the Ostwald ripening of twodimensional (2D) islands ${ }^{4-9}$ and the decay of threedimensional nanostructures on surfaces. ${ }^{10,11}$ Because of the importance of the step energy, considerable attention was paid to theoretical calculations using approximate ${ }^{12-16}$ and first principles methods. ${ }^{17,18}$ Surprisingly, however, no experimental method for the determination of the step energy was known until very recently when three different methods where independently proposed (all from this laboratory). An earlier approach to the problem by Bartelt et al. ${ }^{19}$ made explicit use of the reconstruction induced $C_{2 v}$ symmetry of the $\mathrm{Si}(100)$ surface and is therefore not applicable for surfaces of higher symmetry. The first of the new methods ${ }^{20}$ is based on the observation of the equilibrium shape fluctuations of adatom or vacancy islands. The magnitude of these fluctuations are inversely proportional to the step free energy. The method was applied to vacancy islands on the $\mathrm{Cu}(111)$ surface and a mean step free energy of $\beta a_{\|}=230 \pm 20 \mathrm{meV}$ was derived. ${ }^{20}$ Here and in the following $\beta$ denotes the step free energy per length and $a_{\|}$is the atomic length unit along a densely packed step direction, so that $\beta a_{\|}$is the energy per step atom. The second method ${ }^{21}$ considers the temperature dependence of the equilibrium shape of islands and is based on a theoretical expression for the configuration entropy of the $100 \%$ kinked step. The method was likewise applied to islands on the $\mathrm{Cu}(111)$ surface and a step energy of $\beta a_{\|}$ $=310 \pm 40 \mathrm{meV}$ was reported in the first publication. ${ }^{21}$ Using a larger data set the number reduced to $\beta a_{\|}=270$ $\pm 30 \mathrm{meV} .{ }^{22}$ The third method finally, ${ }^{3}$ is based on the temperature dependence of facet sizes of three-dimensional crystallites. The method appears to be even more demanding on the experiment and the method has not been tested with experimental data so far.

The purpose of this paper is to compare the two methods which make use of 2D-island shapes, both with respect to theoretical as well as to experimental aspects. On the theory side, we point out the principal differences of the energies determined by the two methods and estimate the expected magnitude of the deviation in the obtained values which arises from the differences. On the experimental side, comparison of the two methods is performed by analyzing the same STM images of islands on $\mathrm{Cu}(100), \mathrm{Cu}(111)$, and $\operatorname{Ag}(111)$ (about 13000 islands in total). The main conclusion of the paper is that (i) the principal theoretical differences between the energies determined by the two methods are small compared to the experimental errors and that (ii) the experimental data obtained by the two methods agree quite well.

The paper is organized as follows. The theory of island shape equilibrium fluctuations which was briefly sketched in Ref. 20 is outlined in the following section and it is shown how theory can be employed to extract the step free energy from experimental data on the fluctuations. Section III presents experimental data on the island fluctuations and the data are evaluated in terms of the step energy. The step energies obtained from fluctuations are compared to those obtained from the temperature dependence of the equilibrium shape. In Sec. IV the advantages and disadvantages of the two methods are discussed with respect to each other. Some cumbersome mathematical details of the theory of shape fluctuations are described in the Appendix.

\section{ISLAND SHAPE FLUCTUATIONS}

The relation between the magnitude of island shape fluctuations and the mean step free energy is derived using a capillary mode analysis of Khare and Einstein. ${ }^{23,24}$ Our notation follows largely their treatment and is illustrated in Fig. 1.

The perimeter of an island at time $t$ is described by the radius $r(t, \theta)$. The perimeter of the equilibrium shape of an island $R(\theta)$ is equal to the normalized time average of $r(t, \theta)$, where the normalization ensures that the areas covered by the islands described by $R(\theta)$ and $r(\theta, t)$ are identical. The origin of the angle $\theta$ can be placed at any arbitrary point of the perimeter. Here and in the following we choose the center of the position of minimum curvature as the origin 
(a)

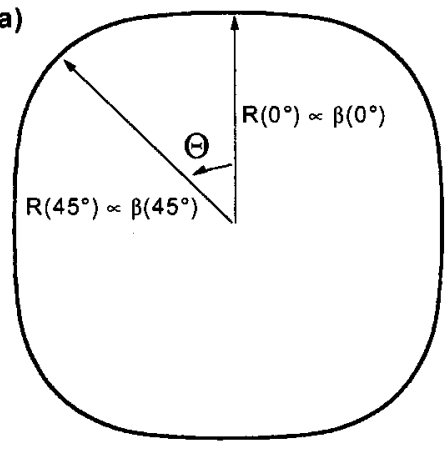

(100)

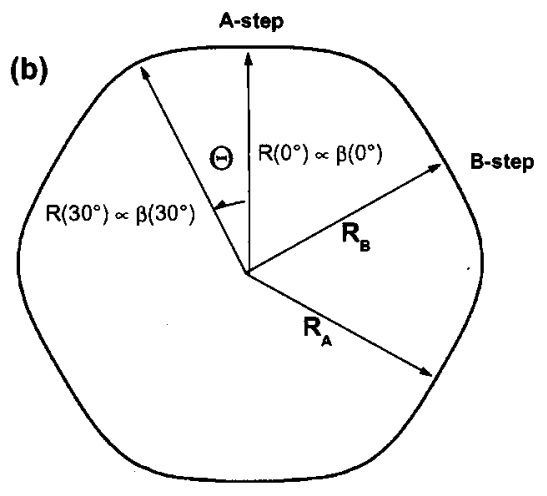

(111)

FIG. 1. Equilibrium shapes of islands on the $\mathrm{Cu}(100)$ and $\mathrm{Ag}(111)$ surfaces around $300 \mathrm{~K}$. For $\mathrm{Ag}(111)$ and $\mathrm{Cu}(111)$ the energies of $A$ steps [displaying a (100) facet] and $B$ steps [displaying a (111) facet] agree within $1 \%$. The island has therefore a nearly hexagonal shape. The perimeter of the equilibrium shape is described by $R(\theta)$.

[in the $A$-step on (111) islands, Fig. 1(b)]. The relative variation $g(\theta, t)$ defined as

$$
g(\theta, t)=\frac{r(\theta, t)-R(\theta)}{R(\theta)}
$$

can be expanded in a Fourier series

$$
g(\theta, t)=\sum_{n} g_{n}(t) \exp (\text { in } \theta)
$$

with $g_{n}(t)=g_{-n} *(t)$ the Fourier coefficients. The experimental data concern the time average of the fluctuation function $G(t)$ defined as

$$
G(t)=\frac{R^{2}}{2 \pi} \int_{0}^{2 \pi} g^{2}(\theta, t) d \theta=R^{2} \sum_{n}\left|g_{n}(t)\right|^{2}
$$

with the mean radius $R$,

$$
R=\frac{1}{2 \pi} \int_{0}^{2 \pi} R(\theta) d \theta
$$

The free total energy of the perimeter is

$$
F(t)=\oint_{r(\theta, t)} \beta d s=\int_{0}^{2 \pi} \beta(\theta) \sqrt{r^{2}(\theta, t)+\left(\frac{\partial r(\theta, t)}{\partial \theta}\right)^{2}} d \theta .
$$

For a given island area, the total free energy is minimal for the equilibrium shape $\left(F \equiv F_{0}\right)$. Because of the fluctuations, the time average of the total free energy is larger by an amount $\langle\Delta F\rangle_{t}$. This deviation can be related to the time average of the Fourier coefficients of the fluctuation function [see the Appendix, Eq. (A13)]. The result is

$$
\begin{aligned}
\langle\Delta F\rangle_{t} & \equiv\left\langle F-F_{0}\right\rangle_{t} \\
& =\langle F\rangle_{t}-F_{0}=\pi \bar{\beta} R \sum_{|n|>1}\left(n^{2}-\alpha\right)\left\langle\left|g_{n}(t)\right|^{2}\right\rangle_{t} .
\end{aligned}
$$

The terms $n= \pm 1$ are omitted from the sum since they correspond to fluctuations of the mean position of the island. Since $r(\theta, t)$ is always measured relative to the center of mass of the islands, island motion is eliminated from the measurement. According to Eq. (6) the contribution of the fluctuations to the free energy is proportional to an average free energy of the step $\bar{\beta}$ defined as

$$
\bar{\beta}=\frac{1}{2 \pi} \int_{0}^{2 \pi} B(\theta) d \theta
$$

with

$$
B(\theta)=\frac{\beta(\theta)}{R} \frac{R^{4}(\theta)}{\left[R^{2}(\theta)+\left(\frac{\partial R(\theta)}{\partial \theta}\right)^{2}\right]^{3 / 2}} .
$$

The number $\alpha$ in Eq. (6) is defined as

$$
\alpha=\frac{1}{2 \pi} \int_{0}^{2 \pi} A(\theta) d \theta
$$

with

$$
A(\theta)=\frac{\beta(\theta) \sqrt{R^{2}(\theta)+\left(\frac{\partial R(\theta)}{\partial \theta}\right)^{2}}}{\bar{\beta} R} .
$$

For circular islands one has $\alpha=1$, but for real equilibrium shapes $\alpha$ deviates slightly from 1 . In the classical continuum limit, each of the capillary modes in Eq. (6) contributes $k_{B} T / 2$ to the free energy, so that

$$
\left\langle\left|g_{n}(t)\right|^{2}\right\rangle_{t}=\frac{k_{B} T}{2 \pi \bar{\beta} R\left(n^{2}-\alpha\right)} .
$$

The time average of the experimental fluctuation function $\langle G(t)\rangle_{t}$ then becomes

$$
\langle G(t)\rangle_{t}=\frac{k_{B} R T}{2 \pi \bar{\beta}} \sum_{|n|>1} \frac{1}{n^{2}-\alpha} .
$$

The sum in Eq. (10) is

$$
\sum_{|n|>1} \frac{1}{n^{2}-\alpha}=\frac{-\Psi(2-\sqrt{\alpha})+\Psi(2+\sqrt{\alpha})}{\sqrt{\alpha}}
$$

with $\Psi(x)$ the derivative of the logarithm of the Gammafunction 


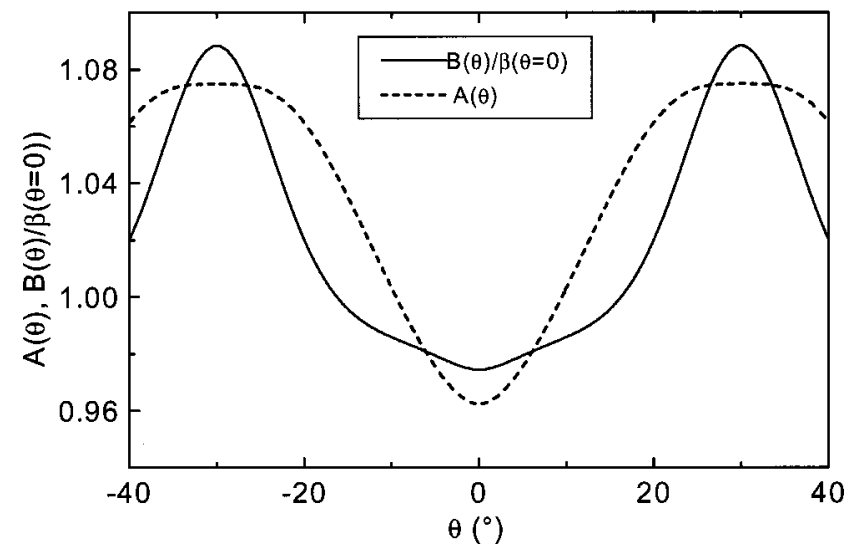

FIG. 2. $A(\theta)$ and $B(\theta) / \beta(\theta=0)$ for the triangular Ising model with an Ising parameter to represent approximately islands on the $\mathrm{Cu}(111)$ surface.

$$
\Psi(x)=\frac{\partial\{\ln [\Gamma(x)]\}}{\partial x} .
$$

For circular islands $(\alpha=1)$ the sum in Eq. (11) is equal to $\frac{3}{2}$ and the magnitude of the fluctuations becomes simply

$$
\langle G(t)\rangle_{t}=\frac{3 k_{B} R T}{4 \pi \bar{\beta}} .
$$

In order to estimate the effect of noncircular shapes of islands on $\alpha$ and the averaging procedure for $\beta$ we have calculated $A(\theta)$ and $B(\theta)$ in the Ising-model ${ }^{25}$ for triangular lattices. The Ising parameter was chosen so that the kink energy is $0.11 \mathrm{eV}$ and the temperature $330 \mathrm{~K}$. The Ising shape is then a rather good representation of the equilibrium shape of islands on $\mathrm{Cu}(111) .^{22}$ The result of the calculation for $A(\theta)$ and $B(\theta) / \beta(\theta=0)$ is shown in Fig. 2. The mean value of $A(\theta)$ [which is equal to $\alpha$, Eq. (8)] is 1.02788 and the mean value of $B(\theta) / \beta(\theta=0)$ is 1.01248 , hence both are close to the result for a circular shape. We therefore conclude that $\bar{\beta}$ defined by Eq. (7) is practically equal to the step free energy at $\theta=0$ and the sum over the capillary modes [Eq. (10)] can be calculated with $\alpha=1$. The step free energy can therefore be obtained using Eq. (13) and experimental data of the time average of the fluctuations $\langle G(t)\rangle_{t}$ as a function of the mean island radius $R$. We note that it is the step free energy $\beta$, or "tension," and not the stiffness $\widetilde{\beta}=\beta$ $+\partial^{2} \beta / \partial \theta^{2}$ which enters Eq. (13). The step stiffness, which provides the restoring force for a local excursion in a step, 7,26 enters when a local description of the fluctuations is given. Here a nonlocal description of the fluctuations appeared to be more convenient, since singularities at $T=0$ are avoided. In a nonlocal description the total (free) energy and its distribution among the modes are considered.

The mean step free energy in Eqs. (7), (13) is the mean free energy at the temperature of measurement. It is therefore useful to discuss briefly the temperature dependence of the step free energy. Since the orientation dependence as a function of temperature is known experimentally from the inverse Wulff construction on the experimental equilibrium shape we can focus on the temperature dependence of the steps oriented along the direction of close packing, the $\langle 011\rangle$ direction. The temperature dependence arises from a configurational and a phonon contribution to the entropy. The phonon contribution is due to the different frequency spectrum of atoms at steps. The magnitude of the contribution can be estimated by assuming that the mean vibrational frequency of an atom is proportional to the square root of its coordination number $C$. This estimate is in agreement with EAM calculations of the phonon contribution to free energies. $^{27,28}$ The configurational contribution is due to the thermal generation of kinks in the steps and can be calculated from the kink energy $\varepsilon_{k} \cdot{ }^{22}$ Thus the temperature dependence is approximately described by

$$
\begin{aligned}
a_{\|} \beta(T)= & a_{\|} \beta(T=0)-2 k_{B} T e^{-\varepsilon_{k} / k_{B} T} \\
& +3 k_{B} T \ln \left(C_{\text {step }} / C_{\text {terrace }}\right)^{1 / 2} .
\end{aligned}
$$

Here, $C_{\text {step }}$ and $C_{\text {terrace }}$ are the coordination numbers of atoms in a step and terrace site, respectively. For $\mathrm{Cu}(111)$ the configurational and phonon contributions to the temperature dependence of the free energy at $300 \mathrm{~K}$ are thus estimated as $-1.03 \times 10^{-2} \mathrm{meV} / \mathrm{K}$ and $-3.25 \times 10^{-2} \mathrm{meV} / \mathrm{K}$, respectively. The temperature dependence of the step free energy is therefore small compared to the absolute value of the step energy (270 meV).

Because of this small temperature dependence, the step free energy can be determined by measuring the fluctuations of islands of different radii at different temperatures. Plotting the results vs. the product of radius $R$ and temperature $T$ [Eq. (13)] facilitates the experiments considerably since the product $R T$ can be varied over a wider range than the radius $R$ for a given temperature. The reason is that for islands grown via homogeneous nucleation at a fixed temperature the mean radius is solely determined by the flux of atoms on the surface, and the radius depends to a low power on the flux $\left(\frac{1}{6}-\frac{1}{4}\right.$ for metal surfaces).

\section{EXPERIMENTAL ANALYSIS OF ISLAND FLUCTUATIONS}

Islands on $\mathrm{Cu}(100), \mathrm{Cu}(111)$, and $\mathrm{Ag}(111)$ were grown by evaporation from a Knudsen cell at temperatures ranging between 280 and $440 \mathrm{~K}$ and the island shapes were observed using a scanning tunneling microscope (STM). For details on the sample preparation as well as on the algorithms used to extract numerical data on the positions of the step edges the reader is referred to Ref. 22. The shape fluctuations were measured by comparing images of the same island obtained in consecutive STM scans. The total time interval between two consecutive images was about $60 \mathrm{~s}$. The shape fluctuations are illustrated in Fig. 3 which displays the mean shapes (full lines) and the actual shapes in two images (squares and circles), for $\mathrm{Cu}(100)$ and $\mathrm{Ag}(111)$, respectively.

Islands decay in size as a function of time because of Ostwald ripening. The islands displayed in Fig. 3 are therefore normalized to the same size. Since the mean fluctuations $\langle G(t)\rangle_{t}$ depends explicitly on the island radius, the shrinking 
(a)

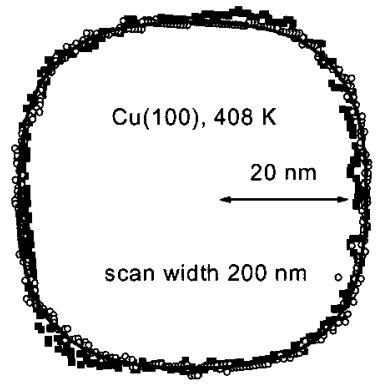

(b)

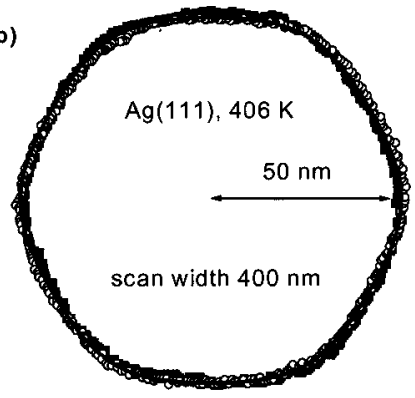

FIG. 3. Mean island shapes (full lines) and individual shapes (circles and squares) of $\mathrm{Cu}(100)$ and $\mathrm{Ag}(111)$. The individual shapes are taken from image Nos. 10 and 50 in the two series which corresponds to a time difference of $2400 \mathrm{~s}$.

size of the islands with time is also of concern for a quantitative determination of the fluctuations. The following procedure was adopted: Individual island shapes were divided into subsets in which the change in size was less than $10 \%$. In each subset, the individual islands were normalized in area to match the mean island area in the set. The fluctuation function $G(t)$ was then calculated from the experimental data on the perimeter for each image according to Eqs. (1), (3) and the resulting values are averaged to obtain an experimental value of $\langle G(t)\rangle_{t}$ for the set. $\langle G(t)\rangle_{t}$ is assigned to the mean radius in the set $R_{s}$ and plotted vs the product of $R_{s}$ and $T$. The final results are displayed in Fig. 4 for $\mathrm{Cu}(111)$ and Figs. 5(a) and 5(b) for $\mathrm{Cu}(100)$ and $\mathrm{Ag}(111)$, respectively. As already noticed by Schlößer et al. ${ }^{20}$ an additional constant term $\left\langle G_{0}\right\rangle$ must be invoked when fitting the experimental data on $\langle G(t)\rangle_{t}$ to a linear dependence on $R_{s} T$ according to Eq. (13)

$$
\langle G(t)\rangle_{t}=\frac{3 k_{B} R T}{4 \pi \bar{\beta}}+\left\langle G_{0}\right\rangle .
$$

The constant term is seen also in our data (Fig. 4) when the data are fitted by a straight line.

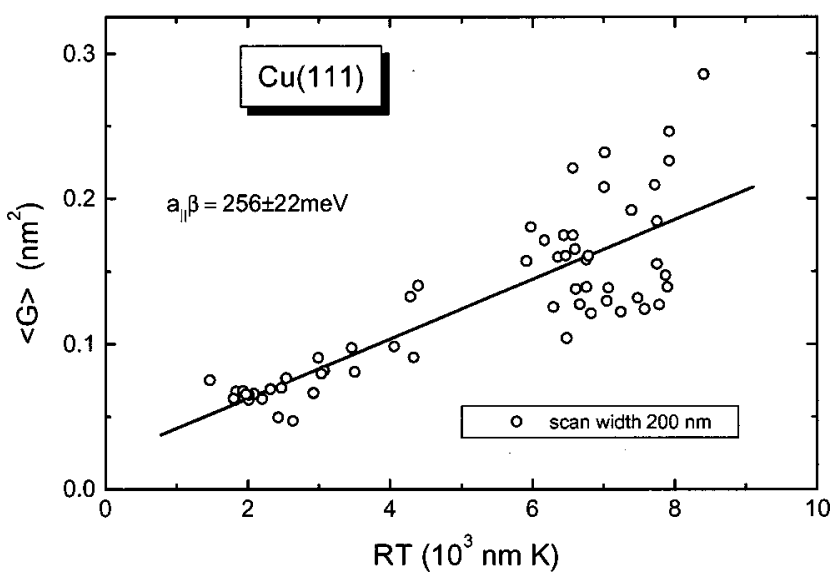

FIG. 4. Mean step fluctuation function $\langle G(t)\rangle_{t}$ for islands on $\mathrm{Cu}(111)$ vs the product of the mean island radius $R$ and temperature $T$.
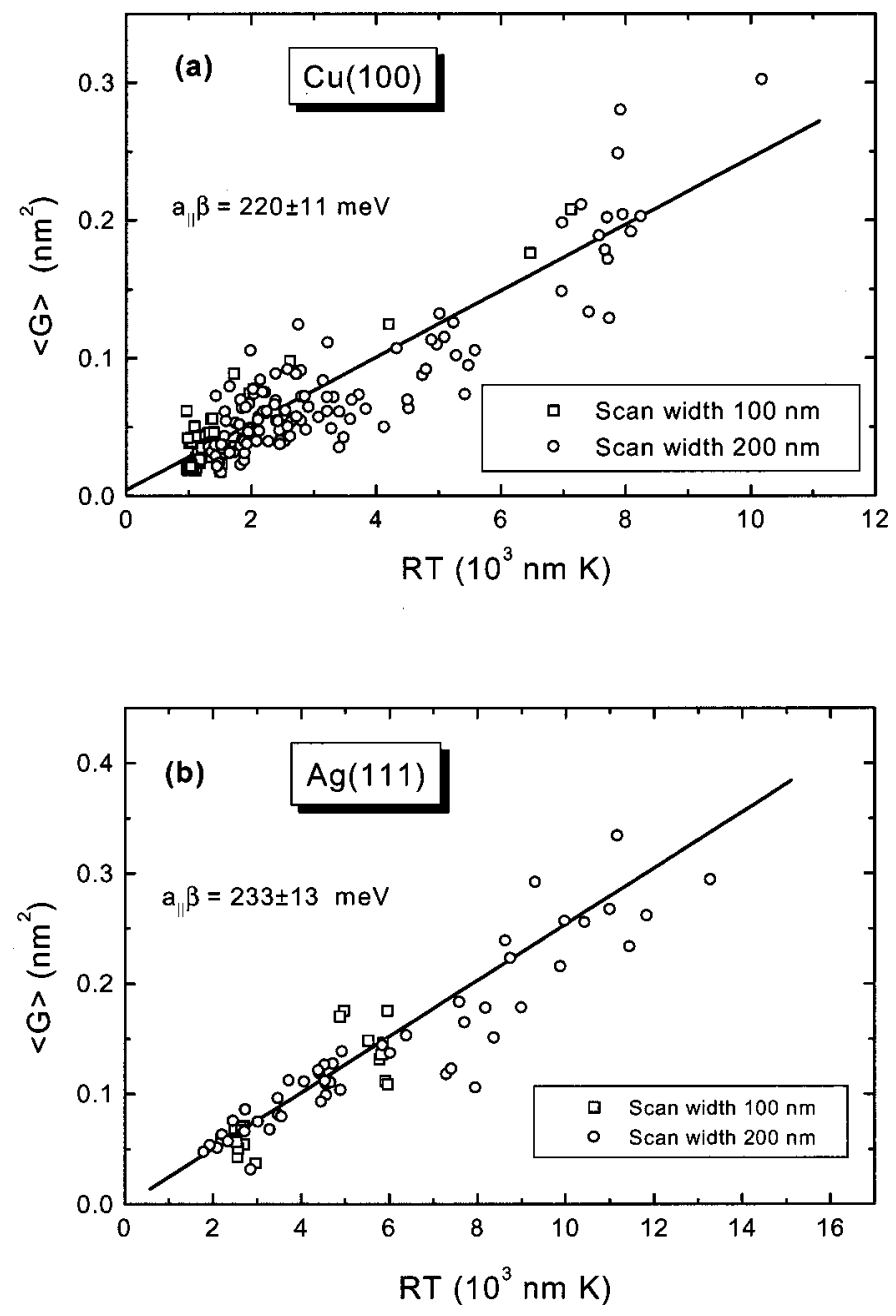

FIG. 5. Mean step fluctuation function $\langle G(t)\rangle_{t}$ for islands on $\mathrm{Cu}(100)$ (a) and $\operatorname{Ag}(111)$ (b) vs the product of the mean island radius $R$ and temperature $T$. A constant term has been subtracted from the data so that $\langle G(t)\rangle_{t}$ fits to a common line (see text for details). The free energy is calculated from the slope according to using Eq. (16).

Schlößer et al. attributed the constant term to the noise originating from the limited pixel resolution of the STM images, and $\left\langle G_{0}\right\rangle$ was determined from the analysis of experimental data obtained at low temperatures. ${ }^{20}$ The proposition that $\left\langle G_{0}\right\rangle$ is mainly due to pixel noise is corroborated by our observation that the constant term obtained from fitting a straight line to the data increases with the scan widths of the STM images. In order to be able to determine the slope from data obtained with different scan widths the constant term was evaluated by fitting a linear slope to the data points obtained for a particular scan width. The constant term was then subtracted from the data and the results for different scan widths were plotted vs $R T$. The data in Figs. 5(a) and 5(b) were treated as described and the line therefore passes nearly through the origin. Nevertheless, the slope was determined from a two-parameter linear regression. The slopes were evaluated according to Eq. (16) and the results for the step mean free energies are displayed in Table I. The errors quoted are of those resulting from the two-parameter fit. The 
TABLE I. Step free energies obtained from the analysis of step fluctuations and from the equilibrium shape method (Ref. 22).

\begin{tabular}{ccc}
\hline \hline Surface & $a_{\|} \beta$ (meV) [fluct.] & $a_{\|} \beta$ (meV) [eq. shape] \\
\hline $\mathrm{Cu}(100)$ & $220 \pm 11$ & $220 \pm 20$ \\
$\mathrm{Cu}(111)$ & $256 \pm 22$ & $270 \pm 30$ \\
$\mathrm{Ag}(111)$ & $233 \pm 13$ & $250 \pm 30$ \\
\hline \hline
\end{tabular}

results are compared to earlier results obtained from the temperature dependence of the equilibrium shape. ${ }^{22}$ We note that for $\mathrm{Cu}(111)$ and $\operatorname{Ag}(111)$ the energies for $A$ and $B$ steps differ only by less than $1 \%$. This difference is neglected in the following discussion.

\section{DISCUSSION}

The step energies obtained from the two entirely different methods agree quite well. In fact, the match is as good as can be expected, considering the statistical errors. Nevertheless, it should be kept in mind that the two methods determine not exactly the same energies. To a good approximation (see discussion in Sec. II), the fluctuation method determines the mean step free energy at the temperature of measurement, while the aspect method determines the energy of the atomically smooth step at $T=0 \mathrm{~K} .^{22}$ The expected differences between the two values can be determined from the phonon and kink contribution to the step free energy [Eq. (14)] on the one hand, and from inverse Wulff plots to the equilibrium shapes on the other. The second column of Table II summarizes the kink energies from Ref. 22. These kink energies are used to calculate the configurational contribution to the free energy of a step oriented along the $\langle 011\rangle$ direction at $350 \mathrm{~K}$. Column 4 estimates the phonon free energy at $350 \mathrm{~K}$ using Eq. (14). The fifth column denoted as $\bar{\beta} / \beta_{0}$ is the ratio of the mean free energy to the free energy of a $\langle 011\rangle$ step at $350 \mathrm{~K}$. The numbers are obtained from inverse Wulf plots to the equilibrium shapes of islands. ${ }^{22}$ In the sixth column denoted as $a_{\|} \beta_{0}(350 \mathrm{~K})$ the free energy of $\langle 011\rangle$ steps is calculated from the experimental data in Table I using the $\bar{\beta} / \beta_{0}$ column. In column 7 these numbers are extrapolated to $T$ $=0 \mathrm{~K}$. These values are then compared to the directly determined experimental data on the step energy at $0 \mathrm{~K}$, as obtained from the analysis of the equilibrium shapes (Table I). The differences between the mean free energies at $350 \mathrm{~K}$ and the step energy at $0 \mathrm{~K}$ is rather small, firstly because the correction terms are small, but secondly, also because averaging over the island perimeter and the finite temperature corrections work in the opposite direction. In general, the step energies at $0 \mathrm{~K}$ agree marginally better than energies directly obtained by the two methods. Considering the error bars that may be fortuitous, however.

We note that the experimental value for the step free energy for $\mathrm{Cu}(111)$ as obtained by the fluctuation method is now larger than the number obtained earlier by Schlößer et al. using the same method $\left[a_{\|} \beta=220 \pm 20 \mathrm{meV}\right.$ (Ref. 20)]. Schlößer et al. investigated the fluctuations of vacancy islands rather than adatom islands as in this work. Though in general, we see no theoretical reason for different step energies of adatom and vacancy islands, a small difference could be present for the smallest islands considered by Schlößer et al. having a radius of $2-3 \mathrm{~nm}$ (8-12 atoms per step). However, the main difference between the result here and in Ref. 20 is attributed to experimental uncertainties. As the data base used in this work is larger $(\sim 2 x)$, the accuracy should be better. Moreover, upon reconsidering the data reported in Ref. 20, and taking into account also the measurement performed at $263 \mathrm{~K}$, which give a larger value for $\left\langle G_{0}\right\rangle$ than the one obtained from a linear regression, it seems that the result $a_{\|} \beta=220 \mathrm{meV}$ (Ref. 20) is too low. Indeed, if the $263 \mathrm{~K}$ results are assumed to be representative for nonfluctuating islands, i.e., for $\langle G\rangle$ at an island radius $R=0$, then a regression of the data of Ref. 20, including the data points measured at $263 \mathrm{~K}$, would give a step free energy of $260 \pm 25$ meV.

Comparison of experimental data to theoretical calculation has already been discussed in Refs. 22 and 29, and the reader is referred to these papers for details. Here, we only mention that the probably only up-to-date first principles calculation concerning step energies on $\mathrm{Cu}(111)$ is in very good agreement with our result. ${ }^{18}$

Finally, we discuss some experimental aspects of the two methods. Both methods provide step energies of comparable accuracy. The slightly larger error for the equilibrium shape method, in particular for the (111) surfaces (Table I) is mostly due to the error in the determination of the kink energy. On the $\mathrm{Cu}(100)$ surface a more accurate value of the kink energy was known from spatial step fluctuations ${ }^{30}$ and the error in the step energy is smaller correspondingly. Both methods have particular advantages and disadvantages and the choice for the optimum method depends on circumstances and the particular interest. If one is interested only in the step free energy as such, the fluctuation method has certain advantages. The method does not require the input of the kink energy and provides sufficiently accurate numbers with a smaller data base than the equilibrium shape method. The

TABLE II. The table summarizes experimental data on steps and presents calculated corrections to make the experimental data on the step energies obtained by the two methods directly comparable. All energies are in meV. See text for further discussion.

\begin{tabular}{lccccccc}
\hline \hline Sample & $\varepsilon_{k}$ & $F_{\text {conf }}$ & $F_{\text {phonon }}$ & $\bar{\beta} / \beta_{0}$ & $a_{\|} \beta_{0}(350 \mathrm{~K})$ & $a_{\|} \beta_{0}(0 \mathrm{~K})_{\text {calc }}$ & $a_{\|} \beta_{0}(0 \mathrm{~K})_{\exp }$ \\
\hline $\mathrm{Cu}(100)$ & 129 & -0.84 & -6.0 & 1.064 & 207 & $213 \pm 11$ & $220 \pm 20$ \\
$\mathrm{Cu}(111)$ & 117 & -1.25 & -11.3 & 1.025 & 250 & $262 \pm 22$ & $270 \pm 30$ \\
$\mathrm{Ag}(111)$ & 101 & -2.1 & -11.3 & 1.025 & 227 & $241 \pm 13$ & $250 \pm 30$ \\
\hline \hline
\end{tabular}


method works with data obtained at a single temperature or in a small temperature range, provided one can generate islands in a sufficiently large size range. This is a definite advantage in particular cases, e.g., when one is interested in the step energies of islands on metals in contact with an electrolyte. On the other hand, the equilibrium shape methods, when applied to a sufficiently large and accurate data set, provides intrinsically more detailed information on energetical aspects. In addition to the step energy at $T=0 \mathrm{~K}$, the method yields the angle dependence of the step free energy at all temperatures. The kink energy is likewise obtained from an Arrhenius plot of the curvature of the steps at the point of minimum curvature. We note that once the step energy is known, from using the fluctuation method, e.g., the kink energy can be determined quite accurately from fitting the minimum curvature on the equilibrium shape at a single temperature. Hence, in some cases a prudent combination of the fluctuation and the equilibrium shape method may be the optimum choice.

\section{APPENDIX}

According to Eq. (1), one can write for the instantaneous radius

$$
r(\theta, t)=R(\theta)[1+g(\theta, t)]
$$

We consider a situation in which the island area is conserved, thus

$$
\int_{0}^{2 \pi} R^{2}(\theta) d \theta=\int_{0}^{2 \pi} r^{2}(\theta, t) d \theta
$$

where $R(\theta)$ is the equilibrium shape of the island, defined by the time average of $r(\theta, t)$

$$
R(\theta)=\sqrt{\left\langle r^{2}(\theta, t)\right\rangle_{t}} .
$$

Inserting Eq. (A1) in the right-hand side of Eq. (A3), one obtains

$$
\langle g(\theta, t)\rangle_{t}=-\frac{1}{2}\left\langle g^{2}(\theta, t)\right\rangle_{t}
$$

Expanding $g(\theta, t)$ in a Fourier series [Eq. (2)] and inserting the expansion in Eq. (A4), one obtains for the Fourier coefficients the expressions

$$
\begin{gathered}
\left\langle g_{0}(t)\right\rangle_{t}+\frac{1}{2}\left\langle g_{0}^{2}(t)\right\rangle_{t}=-\frac{1}{2} \sum_{|n|>0}\left\langle\left|g_{n}(t)\right|^{2}\right\rangle_{t}, \\
\left\langle g_{n}(t)\right\rangle_{t}=-\frac{1}{2} \sum_{i}\left\langle g_{i-n}(t) g_{i}(t)\right\rangle_{t}, \quad n \neq 0 .
\end{gathered}
$$

In this work we consider only small fluctuations $|g(\theta, t)|$ $\ll 1$. Accordingly, we can neglect the term $g_{0}^{2}(\theta, t)$ in Eq. (A5a). The time average of $g_{n}(\theta, t)$ is then given by

$$
\begin{gathered}
\left\langle g_{0}(t)\right\rangle_{t}=-\frac{1}{2} \sum_{|n|>0}\left\langle\left|g_{n}(t)\right|^{2}\right\rangle_{t}, \\
\left\langle g_{n}(t)\right\rangle_{t}=0, n \neq 0
\end{gathered}
$$

because for fluctuation modes $n$ and $m$ which are not correlated $(n, m \neq 0)$, the time averages

$$
\left\langle g_{n}(t) g_{m}(t)\right\rangle_{t} \text { and }\left\langle g_{n}(t) g_{0}(t)\right\rangle_{t} \approx \sum_{|m|>0}\left\langle g_{n}(t) g_{m}^{2}(t)\right\rangle_{t}
$$

are equal to zero.

The free energy of the perimeter of an island is given by Eq. (5). Inserting Eq. (A1) in this expression and making a Taylor expansion in terms of $g(\theta, t)$ and $\partial g(\theta, t) / \partial \theta$ up to second order, one obtains

$$
\begin{aligned}
F(t)= & \int_{0}^{2 \pi} \beta(\theta) \rho(\theta)\left[1+g(\theta, t)+\frac{R^{2}(\theta)}{2 \rho^{2}(\theta)}\right. \\
& \times\left(1-\frac{\left(\frac{\partial R(\theta)}{\partial \theta}\right)^{2}}{\rho^{2}(\theta)}\right) \\
& \left.\times\left(\frac{\partial g(\theta, t)}{\partial \theta}\right)^{2}+\frac{R(\theta)}{\rho^{2}(\theta)} \frac{\partial R(\theta)}{\partial \theta} \frac{\partial g(\theta, t)}{\partial \theta}\right] d \theta
\end{aligned}
$$

or

$$
\begin{aligned}
F(t)= & \int_{0}^{2 \pi} \beta(\theta) \rho(\theta) d \theta+\int_{0}^{2 \pi} \beta(\theta) \rho(\theta) g(\theta, t) d \theta \\
& +R \int_{0}^{2 \pi} \frac{B(\theta)}{2}\left(\frac{\partial g(\theta, t)}{\partial \theta}\right)^{2} d \theta+\int_{0}^{2 \pi} Z(\theta) \frac{\partial g(\theta, t)}{\partial \theta} d \theta
\end{aligned}
$$

where $\rho(\theta)=\sqrt{R^{2}(\theta)+(\partial R(\theta) / \partial \theta)^{2}}$. The first integral in Eq. (A7) is constant with time and represents the step free energy $\beta(\theta)$ integrated along the equilibrium perimeter $R(\theta)$,

$$
F_{0}=\oint_{R(\theta)} \beta(\theta) d s .
$$

Because $B(\theta)$ and $(\partial g(\theta, t) / \partial \theta)^{2}$ are both positive for all $\theta$, one can write the third integral of Eq. (A7) as

$$
\int_{0}^{2 \pi} \frac{B(\theta)}{2}\left(\frac{\partial g(\theta, t)}{\partial \theta}\right)^{2} d \theta=\frac{\bar{\beta} R}{2} \int_{0}^{2 \pi}\left(\frac{\partial g(\theta, t)}{\partial \theta}\right)^{2} d \theta
$$

with

$$
\begin{gathered}
\min [B(\theta)] \leqslant \bar{\beta} \leqslant \max [B(\theta)], \\
\bar{\beta} \approx \frac{1}{2 \pi} \int_{0}^{2 \pi} B(\theta) d \theta, \\
B(\theta)=\frac{\beta(\theta) R^{2}(\theta)}{R \rho(\theta)}\left(1-\frac{\left(\frac{\partial R(\theta)}{\partial \theta}\right)^{2}}{\rho^{2}(\theta)}\right),
\end{gathered}
$$


where $R$ is the mean island radius [Eq. (4)]. Replacing $g(\theta, t)$ by the Fourier expansion of this function and performing the integral, we obtain for the time average of the third integral of Eq. (A7):

$$
\left\langle\int_{0}^{2 \pi} \frac{B(\theta)}{2}\left(\frac{\partial g(\theta, t)}{\partial \theta}\right)^{2} d \theta\right\rangle_{t}=\pi \bar{\beta} R \sum_{n} n^{2}\left\langle\left|g_{n}(t)\right|^{2}\right\rangle_{t} .
$$

The time average of the second and fourth integral of Eq. (A7) can be evaluated by noting that

$$
\langle g(\theta, t)\rangle_{t}=\sum_{n}\left\langle g_{n}(t)\right\rangle_{t} e^{i n \theta}=\left\langle g_{0}(t)\right\rangle_{t},
$$

because the nonzero order terms in the sum vanish [Eq. (A6)]. The zero order term in the Fourier expansion is

$$
\left\langle g_{0}(t)\right\rangle_{t}=-\frac{1}{2} \sum_{|n|>0}\left\langle\left|g_{n}(t)\right|^{2}\right\rangle
$$

[Eq. (A6)] and does not depend on $\theta$. Thus

$$
\left\langle\frac{\partial g(\theta, t)}{\partial \theta}\right\rangle_{t}=\left\langle\frac{\partial g_{0}(t)}{\partial \theta}\right\rangle_{t}=0
$$

and the time average of the fourth integral of Eq. (A7) vanishes. The time average of the second integral becomes

$$
\begin{aligned}
& \left\langle\int_{0}^{2 \pi} \beta(\theta) \rho(\theta) g(\theta, t) d \theta\right\rangle_{t} \\
& =-\int_{0}^{2 \pi} \frac{\beta(\theta) \rho(\theta)}{2} \sum_{|n|>0}\left\langle\left|g_{n}(t)\right|^{2}\right\rangle_{t} d \theta \\
& =\pi \bar{A} \sum_{|n|>0}\left\langle\left|g_{n}(t)\right|^{2}\right\rangle_{t}
\end{aligned}
$$

where $\min [\beta(\theta) \rho(\theta)] \leqslant \bar{A} \leqslant \max [\beta(\theta) \rho(\theta)]$ or

$$
\bar{A} \approx \frac{1}{2 \pi} \int_{0}^{2 \pi} \beta(\theta) \rho(\theta) d \theta .
$$

Since the zero order term in Eq. (A10) does not contribute to the integral $(n=0)$ we can combine this equation with Eq. (A11), resulting in

$$
\langle F(t)\rangle_{t}=\pi \bar{\beta} R \sum_{|n|>0}\left(n^{2}-\alpha\right)\left\langle\left|g_{n}(t)\right|^{2}\right\rangle_{t},
$$

where

$$
\alpha=\frac{\bar{A}}{\bar{\beta} R} .
$$

Above we defined the equilibrium shape of an island $R(\theta)$ through Eq. (A3). Instead of this, one could define an "average island shape" $R^{\#}(\theta)$ as $R^{\#}(\theta)=\langle r(\theta, t)\rangle_{t}$ and use this to derive an expression for the free energy of the island perimeter. For the relative variation

$$
g^{\#}(\theta, t)=\frac{r(\theta, t)-R^{\#}(\theta)}{R^{\#}(\theta)},
$$

Eqs. (A4)-(A6) do not apply, but one can easily show that

$$
\left\langle g^{\#}(\theta, t)\right\rangle_{t}=0 .
$$

This means that the Fourier coefficients $\left\langle g_{n}^{\#}(t)\right\rangle_{t}$ are equal to zero or $\left\langle g_{n}^{\#}(t)\right\rangle_{t}=\left\langle g_{n}(t)\right\rangle_{t}=0$ for $n \neq 0$. Thus, only the zero order Fourier coefficients $\left\langle g_{n}^{\#}(t)\right\rangle_{t}$ and $\left\langle g_{0}(t)\right\rangle_{t}$ are different. Taking this into account in the evaluation of the Taylor expansion Eq. (A7) for the free energy of the island perimeter, one gets essentially the same expression Eq. (A13) for $\langle F(t)\rangle_{t}$, but $\alpha$ has to be replaced by $\alpha^{\#}=0$ and $\bar{\beta}^{\#}$ is given by Eqs. (A8) and (A9) with $R(\theta)$ replaced by $R^{\#}(\theta)$.
*Corresponding author, Email address: h.ibach@fz-juelich.de

${ }^{1}$ C. Jayaprakash and W. F. Saam, Phys. Rev. B 30, 3916 (1984).

${ }^{2}$ M. Wortis, in Chemistry and Physics of Solid Surfaces, edited by

R. Vanselow and R. Howe (Springer, New York, 1988), Vol. 7, p. 367.

${ }^{3}$ H. P. Bonzel and A. Emundts, Phys. Rev. Lett. 84, 5804 (2000).

${ }^{4}$ J. B. Hannon et al., Phys. Rev. Lett. 79, 2506 (1997).

${ }^{5}$ K. Morgenstern, Phys. Rev. Lett. 80, 556 (1998).

${ }^{6}$ G. S. Icking-Konert, M. Giesen, and H. Ibach, Surf. Sci. 398, 37 (1998).

${ }^{7}$ M. Giesen and G. S. Icking-Konert, Surf. Sci. 412/413, 645 (1998).

${ }^{8}$ W. Theis, N. C. Bartelt, and R. M. Tromp, Phys. Rev. Lett. 75, 3328 (1995).

${ }^{9}$ N. C. Bartelt, W. Theis, and R. M. Tromp, Phys. Rev. B 54, 11741 (1996).

${ }^{10}$ M. Giesen and H. Ibach, Surf. Sci. 431, 109 (1999).

${ }^{11}$ M. Giesen, G. S. Icking-Konert, and H. Ibach, Phys. Rev. Lett. 80, 552 (1998)

${ }^{12}$ C.-L. Liu and J. B. Adams, Surf. Sci. 294, 211 (1993).

${ }^{13}$ Z.-J. Tian and T. S. Rahman, Phys. Rev. B 47, 9751 (1993).
${ }^{14}$ R. C. Nelson et al., Surf. Sci. 295, 462 (1993).

${ }^{15}$ P. Stoltze, J. Phys.: Condens. Matter 6, 9495 (1994).

${ }^{16}$ S. V. Khare and T. L. Einstein, Surf. Sci. 314, L857 (1994).

${ }^{17}$ P. J. Feibelman, Phys. Rev. B 52, 16845 (1995).

${ }^{18}$ P. Feibelman, Phys. Rev. B 60, 11118 (1999).

${ }^{19}$ N. C. Bartelt, R. M. Tromp, and E. D. Williams, Phys. Rev. Lett. 73, 1656 (1994).

${ }^{20}$ D. C. Schlößer et al., Phys. Rev. Lett. 82, 3843 (1999).

${ }^{21}$ G. S. Icking-Konert, M. Giesen, and H. Ibach, Phys. Rev. Lett. 83, 3880 (1999).

${ }^{22}$ M. Giesen, C. Steimer, and H. Ibach, Surf. Sci. 471, 80 (2001).

${ }^{23}$ S. V. Khare and T. L. Einstein, Phys. Rev. B 54, 11752 (1996).

${ }^{24}$ S. V. Khare and T. L. Einstein, Phys. Rev. B 57, 4782 (1998).

${ }^{25}$ R. K. P. Zia, J. Stat. Phys. 45, 801 (1986).

${ }^{26}$ N. C. Bartelt et al., Phys. Rev. B 48, 15453 (1993).

${ }^{27}$ A. Kara, S. Durukanoglu, and T. S. Rahman, Phys. Rev. B 53, 15489 (1996).

${ }^{28}$ A. Kara, S. Durukanoglu, and T. S. Rahman, J. Chem. Phys. 106, 2031 (1997).

${ }^{29}$ M. Giesen, Prog. Surf. Sci. 68, 1 (2001).

${ }^{30}$ M. Giesen-Seibert et al., Surf. Sci. 329, 47 (1995). 